\title{
Caracterización de trabajadores sexuales del área norte de Santiago portadoras de Chlamydia trachomatis.
}

Cristián Pizarro V1, Fernando Pérez $\mathrm{C}^{2}$, Raúl de la Fuente $\mathrm{E}^{3}$, Ester Santander $\mathbf{C}^{4}$.

'Dermatólogo, Unidad de Dermatología, Hospital de Purranque, Osorno; 'Departamento de Dermatología. Facultad de Medicina, Universidad de Chile; ${ }^{3}$ Facultad de Medicina, Universidad de Chile; ${ }^{4}$ Unidad de Atención y Control en Salud Sexual (UNACESS). Servicio de Dermatología, Hospital San José.

\section{Resumen}

Marco Teórico: Chlamydia trachomatis, agente bacteriano de infección de transmisión sexual (ITS) más frecuente en el mundo, se asocia a patología inflamatoria y neonatal, y a infertilidad. Las trabajadoras sexuales constituyen un grupo de alto riesgo para su contagio. Objetivo: Caracterizar trabajadoras sexuales portadoras de $C$. trachomatis del área norte de Santiago. Pacientes y Métodos: 36 trabajadoras sexuales con inmunofluorescencia directa positiva para C. trachomatis controladas en una Unidad de ITS. Se analizó en sus fichas clínicas: nivel educacional, uso de alcohol y drogas, tendencia sexual, ITS asociadas y uso de preservativo. Esta información se comparó con fichas clínicas de trabajadoras sexuales no portadoras. Resultados: No se encontraron diferencias estadísticamente significativas entre portadoras y no portadoras, a partir de la revisión de registros clínicos. Conclusión: $E$ l diagnóstico de infección de $C$. trachomatis y su adecuado control requieren de procedimientos estandarizados para su implementación en grupos de alto riesgo.

Palabras claves: chlamydia trachomatis, infección de transmisión sexual, trabajadoras sexuales.

\section{Summary}

Background: Chlamydia trachomatis, the most frequent bacterial agent of sexually transmited infections (STI) in the world, is associated with inflammatory and neonatal diseases, as well as with infertility. Sex workers are a high risk group. Objetive: To characterize sex workers in the northern area of Santiago, who are C. trachomatis carriers. Patients and Methods: 36 sex workers, whose direct immunofluorescence assays tested positive for $C$. trachomatis, attending a STI Unit. The following information was analyzed from their clinical records: educational level, alcohol and drugs use, sexual tendency, associated STIs and condom use. It was compared to the information from clinical records of non C. trachomatis carrier sex workers. Results: No statistically significant difference between carriers and noncarriers was found, regarding the aforementioned features, from the clinical records review. Conclusion: The diagnosis of $C$. trachomatis infection and its proper control require standardized proceedings to be implemented in high risk groups.

Key words: chlamydia, workers, trachomatis, sexual trasmitted infeccions, sex.

\section{Introducción}

Chlamydia trachomatis es la bacteria de transmisión sexual más frecuente en el mundo'. Corresponde a una bacteria intracelular obligada, agente de gran importancia en el desarrollo de infecciones de transmisión sexual (ITS) en todo el mundo. La principal proteína de la membrana externa posee más de 20 serotipos, siendo los más frecuentes aquellos entre el $\mathrm{D}$ y el $\mathrm{K}$ en la patogenia de las ITS². En Estados Unidos, se describen al menos 4 millones de nuevos casos por año ${ }^{3}$. En Chile, la prevalencia registrada en mujeres atendidas en centros ginecológicos, tanto privados como públicos, mediante reacción en cadena de la polimerasa (PCR) y muestras endo-cervicales obtenidas por tórula de algodón, ha sido de 4,7\% ${ }^{4}$. Ingresa por microabrasiones de la piel o mucosa en el acto sexual proveniente de secreción de la pareja, y tiene alta transmisibilidad, lo que facilita la infección asintomática. Si bien esta infección no conlleva riesgo vital, su relevancia está determinada por la capacidad de este agente de estimular la producción persistente de citoquinas pro-inflamatorias, lo que puede llevar a daño permanente en los tejidos infectados ${ }^{5}$. Chlamydia trachomatis se asocia al $50 \%$ de los procesos inflamatorios pélvicos, pudiendo causar además endometritis, salpingitis, peritonitis pelviana, embarazo ectópico e infertilidad; constituye además la principal causa de uretritis no gonocócica

Correspondencia: Raúl de la Fuente

Correo electrónico: rauldelaes@yahoo.es 
en hombres y afecta a más del $50 \%$ de los neonatos expuestos, siendo la conjuntivitis y la neumonitis las entidades más importantes ${ }^{6}$. El método diagnóstico más utilizado es a través de muestras endocervicales obtenidas por tórula de algodón. Sin embargo, deben ser tomadas por un profesional capacitado, lo que dificulta la realización de tamizaje como estrategia de control. Un método alternativo es el estudio microbiológico muestras de orina, que pueden ser recolectadas por el mismo paciente ${ }^{7}$. En los últimos años, las técnicas de amplificación de ácidos nucleicos, como la reacción en cadena de la polimerasa (PCR) han demostrado alta sensibilidad ${ }^{8,9}$. En nuestro medio, la confirmación se realiza mediante la visualización de anticuerpos a través de inmunofluorescencia directa (IFD), a partir de muestras endocervicales en pacientes con sospecha.

Las trabajadoras sexuales constituyen un grupo de especial riesgo de portación y transmisión de esta patología, debido a las condiciones en las que desarrollan su actividad, uso intermitente de preservativo, y en ocasiones, asistencia irregular a controles de salud sexual ${ }^{10}$. Si bien las trabajadoras sexuales chilenas están más conscientes de los riesgos inherentes a su actividad, hay un importante número de extranjeras ejerciendo trabajo sexual que no realiza sus controles ni tienen educación en uso de preservativos. La inmigración creciente pondrá en evidencia la necesidad de aumentar la cobertura y vigilar permanentemente la evolución de las ITS. Se estima en cerca de un $50 \%$ la cobertura de los controles de salud sexual de estas trabajadoras en el área norte de Santiago.

\section{Pacientes y métodos}

Se revisaron las fichas de todas las trabajadoras sexuales que acudieron a control en la Unidad de ITS del Hospital San José de Santiago durante el año 2014. A partir de estos documentos, se efectuó un registro de todas aquellas pacientes dedicadas a esta actividad que tuvieran frotis endocervical con IFD positiva para Chlamydia tracomatis, y se extrajo información de este grupo en relación a los siguientes aspectos: nivel educacional, uso de alcohol y drogas, tendencia sexual, ITS asociada (serología para virus hepatitis $\mathrm{B}$ y VIH, y VDRL) y uso de preservativo, tanto con sus clientes como con sus respectivas parejas sexuales estables. Las fichas de las pacientes fueron revisadas individualmente, para cotejar los datos aparecidos en las cartolas de seguimiento, tanto con las respectivas fichas clínicas, como con los formularios de registro de enfermedades de notificación obligatoria (ENO). Cada uno de los parámetros evaluados se compararon con la información obtenida en trabajadoras sexuales del área norte de Santiago con IFD negativa para Chlamydia tracomatis. El análisis estadístico se realizó mediante la prueba

\begin{tabular}{|l|c|c|}
\hline & PORTADORAS & $\begin{array}{c}\text { NO } \\
\text { PORTADORAS }\end{array}$ \\
\hline Nivel educacional & $\mathbf{n}$ & $\mathbf{n}$ \\
\hline Básica & 7 & 30 \\
\hline Media & 21 & 81 \\
\hline Superior & 4 & 17 \\
\hline Analfabeta & 1 & 1 \\
\hline Desconocido & 3 & 1 \\
\hline TOTAL & 36 & 130 \\
\hline
\end{tabular}

Tabla 1. Nivel educacional de trabajadoras sexuales portadoras y no portadoras de $\mathrm{C}$. trachomatis.

de chi cuadrado. En el caso de la cormobilidad asociada (ITS), la información se describe como porcentajes.

\section{Resultados}

Se revisó un total de 36 fichas correspondientes a trabajadoras sexuales del área norte de Santiago que obtuvieron IFD positiva para $\mathrm{C}$. trachomatis, resultando todas de sexo femenino, 26 chilenas $(72,2 \%)$ y 10 extranjeras (27,8\%). La edad promedio de las pacientes en estudio fue de $34,9 \pm 10,7$ años. Para ser incluida, las pacientes debían ser trabajadoras sexuales portadoras de C. trachomatis y acudir a control periódico de salud. No se realizó otra distinción en relación a su situación de salud, ya que otras variables tanto demográficas como epidemiológicas son precisamente parte de la caracterización que se pretende mediante este estudio.

Al analizar el nivel educacional de las pacientes portadoras, se tiene que en la mayor parte de los casos, estas han completado la escolaridad básica y media, observándose esta situación es más de la mitad de los sujetos de estudio. Si bien esta situación se repite en las trabajadoras sexuales no portadoras, no se encontró diferencia estadísticamente significativa entre ambos grupos, lo que descarta la relación entre nivel educacional y portación de la infección (P-value > 0,05) (Tabla 1).

Se pesquisó además el consumo de alcohol y drogas, ya que el uso de estas sustancias se asocia a mayor riesgo de contraer ITS ${ }^{11}$. Se declaró consumo de alcohol por parte de dos tercios de las trabajadoras sexuales con IFD positiva para C. trachomatis (24 de 36). Sin embargo, entre aquellas pacientes con IFD negativa, 95 de ellas declara el mismo hábito. El análisis estadístico descarta diferencia significativa entre ambos grupos (P-value > 0,05) (Tabla 2). 


\begin{tabular}{|l|c|c|}
\hline & PORTADORAS & $\begin{array}{c}\text { NO } \\
\text { PORTADORAS }\end{array}$ \\
\hline $\begin{array}{l}\text { Consumo de } \\
\text { alcohol }\end{array}$ & $\mathrm{n}$ & $\mathrm{n}$ \\
\hline Sí & 24 & 95 \\
\hline No & 9 & 30 \\
\hline Sin información & 3 & 5 \\
\hline TOTAL & 36 & 130 \\
\hline
\end{tabular}

Tabla 2. Consumo de alcohol en trabajadoras sexuales portadoras y no portadoras de C. trachomatis.

En relación al consumo de drogas, aproximadamente un 19,4\% de las trabajadoras sexuales portadoras (7 de 36) admite este hábito. Entre las no portadoras, un 21,5\% (28 de 130) también refiere esta práctica. Esta similitud se constata en el análisis estadístico, que descarta diferencia significativa ( $P$-value $>0,05$ ) (Tabla 3).

Por otra parte, se categorizó a las pacientes según la tendencia sexual reportada. Entre las portadoras, un 94,4 \% (34 de 36) refiere practicar únicamente relaciones sexuales heterosexuales. Un 5,6 \% no consignó tendencia sexual. Ninguna de ellas declaró ser homosexual o bisexual. Entre las no portadoras, un 93,8 \% (122 de 130) es heterosexual. El análisis estadístico descarta diferencia entre portadoras y no portadoras ( $\mathrm{P}$-value > 0,05) (Tabla 4).

Respecto a la presencia de ITS concomitantes, se indagó la coexistencia de sífilis, hepatitis B e infección por VIH. De la población portadora, en estudio, solo se encontró el antecedente de VDRL reactivo en 7 de 36 casos (19,4\%). No se encontraron casos de coinfección por virus hepatitis B o VIH. Entre las no portadoras, si bien la proporción de VDRL reactivos es menor (10 de 130 pacientes, $7,7 \%)$, también hay 1 caso de serología positiva para VHB $(0,76$ \%) y 2 portadoras de $\mathrm{VIH}(1,5 \%$ ) (Tabla 5).

Otra variable estudiada fue el uso de preservativo por parte de las trabajadoras sexuales. Dado que esta práctica constituye la medida más importante para evitar la transmisión de las ITS, se investigó el uso tanto con sus clientes como con sus parejas estables. En primer lugar, el uso de condón con los clientes fue referido en proporciones similares en ambos grupos de trabajadoras sexuales, con un $83,3 \%$ de las portadoras (30 de 36) y un $89,2 \%$ de las no portadoras (116 de 130) que señalan utilizarlo siempre 0 a veces. Esta semejanza se ve refrendada en el análisis estadístico, que descarta diferencias significativas entre ambos grupos (P-value $>0,05)$ (Tabla 6).

\begin{tabular}{|l|c|c|}
\hline & PORTADORAS & $\begin{array}{c}\text { NO } \\
\text { PORTADORAS }\end{array}$ \\
\hline $\begin{array}{l}\text { Consumo de } \\
\text { drogas }\end{array}$ & $\mathbf{n}$ & $\mathbf{n}$ \\
\hline Sí & 7 & 28 \\
\hline No & 26 & 98 \\
\hline Sin información & 3 & 4 \\
\hline TOTAL & 36 & 130 \\
\hline
\end{tabular}

Tabla 3. Consumo de drogas en trabajadoras sexuales portadoras y no portadoras $\mathrm{C}$. trachomatis.

\begin{tabular}{|l|c|c|}
\hline Tendencia sexual & PORTADORAS & $\begin{array}{c}\text { NO } \\
\text { PORTADORAS }\end{array}$ \\
\hline Heterosexual & 34 & 122 \\
\hline Homosexual & 0 & 2 \\
\hline Bisexual & 0 & 1 \\
\hline No informado & 2 & 5 \\
\hline TOTAL & 36 & 130 \\
\hline
\end{tabular}

Tabla 4. Tendencia sexual reportada en trabajadoras sexuales portadoras y no portadoras de $\mathrm{C}$. trachomatis.

\begin{tabular}{|l|c|c|}
\hline ITS pesquisada & $\mathbf{n}$ & porcentaje \\
\hline VDRL reactivo & 7 & $19,4 \%$ \\
\hline VHB positivo & 0 & $0 \%$ \\
\hline VIH positivo & 0 & $0 \%$ \\
\hline
\end{tabular}

Tabla 5. Infecciones de transmisión sexual (ITS) concomitantes pesquisadas trabajadoras sexuales portadoras de $\mathrm{C}$. trachomatis.

\begin{tabular}{|l|c|c|}
\hline & PORTADORAS & $\begin{array}{c}\text { NO } \\
\text { PORTADORAS }\end{array}$ \\
\hline $\begin{array}{l}\text { Uso preservativo } \\
\text { con clientes }\end{array}$ & $\mathrm{n}$ & $\mathrm{n}$ \\
\hline Siempre & 24 & 91 \\
\hline A veces & 6 & 25 \\
\hline Nunca & 4 & 8 \\
\hline No informado & 2 & 6 \\
\hline TOTAL & 36 & 130 \\
\hline
\end{tabular}

Tabla 6. Uso de preservativo con clientes en trabajadoras sexuales portadoras y no portadoras de $\mathrm{C}$. trachomatis. 


\begin{tabular}{|l|c|c|}
\hline & PORTADORAS & $\begin{array}{c}\text { NO } \\
\text { PORTADORAS }\end{array}$ \\
\hline $\begin{array}{l}\text { Uso preservativo } \\
\text { con pareja }\end{array}$ & $\mathrm{n}$ & $\mathrm{n}$ \\
\hline Siempre & 4 & 11 \\
\hline A veces & 3 & 26 \\
\hline Nunca & 21 & 62 \\
\hline No informado & $\mathbf{8}$ & $\mathbf{3 1}$ \\
\hline
\end{tabular}

Tabla 7. Uso de preservativo con pareja estable en trabajadoras sexuales portadoras y no portadoras de $\mathrm{C}$. trachomatis.

Por último, se investigó acerca del uso de preservativo por parte de las trabajadoras sexuales con sus parejas estables. Se observó que un $19,2 \%$ de las portadoras (7 de 36 ) y un $28,4 \%$ de las no portadoras (37 de 130) refiere tener sexo con protección siempre 0 a veces. Estas proporciones no arrojan diferencia estadísticamente significativa (P-value $>0,05)$ (Tabla 7).

\section{Discusión}

A pesar de ser por definición un grupo de riesgo de transmisión de ITS, las trabajadoras sexuales activas en Chile constituyen un grupo escasamente estudiado a la fecha. En un primer estudio que busca relacionar este grupo con la portación de Chlamydia trachomatis, la infección bacteriana más común, no se encontraron diferencias que permitan establecer con claridad factores de riesgo para el contagio. Es necesario establecer, en primer lugar, que la caracterización realizada en este estudio solo se centra en las trabajadoras sexuales. Los usuarios de trabajadoras sexuales constituyen un grupo de riesgo debido al elevado número de parejas sexuales que suelen tener (rara vez visitan a una sola trabajadora), a la gran dificultad que supone realizar una investigación sistematizada en este grupo de individuos (muchos de ellos ocultan este hábito y no suelen acudir a médico salvo en caso de presentar manifestaciones clínicas de alguna ITS) y al hecho de que en muchos casos tienen una pareja estable con la que suelen tener actividad sexual sin protección, transmitiendo de este modo ITS que pueden permanecer asintomáticas por largos períodos sin que la persona afectada tenga conocimiento, como sucede en la infección por $\mathrm{C}$. trachomatis. Si bien no se encontraron diferencias entre trabajadoras sexuales portadoras y no portadoras para aquellas conductas que puedan suponer factores de riesgo (uso de alcohol y drogas, uso de preservativo), es posible que dicha diferencia esté dada por características de sus clientes, en términos de la portación por parte de estos $\mathrm{C}$. trachomatis u otros patógenos de transmisión sexual, o de sus hábitos. Estudios actuales avalan esta teoría, ya que se ha documentado una mayor prevalencia de ITS en mujeres que han declarado tener sexo con individuos que consumen drogas, con individuos que pagan por sexo, con compañeros sexuales desconocidos o que han tenido sexo bajo los efectos de las drogas o el alcohol ${ }^{12}$. Todas las situaciones descritas son más comunes en las trabajadoras sexuales que en las mujeres que no realizan esta actividad, lo que pone a este grupo en una situación de mayor riesgo.

En relación con lo anterior, la literatura actual señala que la pesquisa centrada únicamente en lesiones de localización urogenital es insuficiente para el adecuado diagnóstico de infección por C. trachomatis, estando ampliamente documentada la existencia de casos positivos de localización orofaringea o rectal, con negatividad de la respectiva muestra urogenital, lo que hubiera significado un fracaso en la pesquisa ${ }^{13,14}$. En consecuencia, otra posible explicación para la portación por parte de las trabajadoras sexuales puede estar determinada por el tipo de relaciones sexuales solicitadas, o del grado de riesgo que conlleven, como por ejemplo, el sexo anal u oral con o sin preservativo. Considerando que no se encontró diferencia estadísticamente significativa para el uso de preservativo entre portadoras y no portadoras, ya sea con sus clientes o con sus parejas estables, el conocimiento detallado acerca de este aspecto, o bien la extracción de muestras no solo urogenitales, sino además podría ser importante en determinar factores que permitan explicar por qué algunas trabajadoras sexuales portan C. trachomatis y otras no.

Por otra parte, los resultados obtenidos son consistentes con lo documentado en la literatura, que establece que el reporte verbal de actividades sexuales de riesgo, o la presencia de manifestaciones clínicas de enfermedad, son por sí solas insuficientes para el diagnóstico de ITS, y que la combinación de ambas, en el caso de la infección por C. trachomatis, ha permitido la pesquisa de compromiso tanto urogenital como extragenital en mujeres de alto riesgo que consultan en policlínicos de ITS, como lo son las pacientes estudiadas ${ }^{15,16}$. Futuros estudios, basados tanto en entrevistas a las pacientes como en la realización de un examen físico completo, podrían ser de utilidad para determinar variables que permitan establecer diferencias entre mujeres portadoras y no portadoras de este agente.

Por último, cabe señalar la importancia de establecer protocolos de pesquisa estandarizados, dirigidos a controlar de mejor manera esta infección utilizando técnicas de confirmación diagnóstica ya disponibles en nuestro medio como la IFD, los que si bien serían demasiado costosos de implementar en la población general, sí serían de utilidad al estar enfocados en grupos de riesgo, como las trabajadoras sexuales. De este 
modo, también sería posible reducir de un modo importante el impacto de los problemas de salud asociados a ella, los que afectan aspectos tan significativos como la vida sexual o la planificación familiar. El logro de este objetivo estaría ayudado por la baja resistencia a antibióticos que, a la fecha, se ha descrito para C. trachomatis en la literatura tanto in vivo como in vitro, aun en países con alta tasa de uso de antibióticos, o tras su uso masivo ${ }^{17,18}$. Por lo tanto, el beneficio de implementar políticas destinadas al diagnóstico y tratamiento de esta ITS parece factible en las trabajadoras sexuales, incluso en las extranjeras, sin perjuicio del perfil de uso o resistencia a antibióticos de sus países de origen.

En conclusión, el presente trabajo es un primer estudio acerca de la situación actual en Chile de población de alto riesgo portadora de la ITS bacteriana más frecuente a nivel mundial. A partir de esta caracterización, no se logró encontrar elementos demográficos, culturales o conductuales que permitan establecer factores de riesgo que distingan a las portadoras de las no portadoras. A la espera de futuros estudios que permitan determinar dichos factores de riesgo para a continuación plantear políticas educativas a nivel comunitario, se realza por mientras el rol fundamental del dermatólogo en una anamnesis, pero fundamentalmente, en un examen físico completo que descarte todas las posibles ubicaciones anatómicas de las lesiones asociadas, lo que contribuye a un mejor aprovechamiento de las pruebas diagnósticas a disposición en los Servicios de Salud, posibilitando con ello una mejor cobertura en el tratamiento y con ello, limitando la aparición de complicaciones secundarias o secuelas.

\section{Referencias bibliográficas}

1. Global incidence and prevalence of selected curable sexually transmitted infections-2008. WHO 2012. http://apps.who.int/iris/ bitstream/10665/75181/1/9789241503839_eng.pdf.

2. Frej-Mądrzak M, Teryks-Wołyniec D, Jama-Kmiecik A, Sarowska J, ChoroszyKról I. Diagnosing Chlamydia Trachomatis Urinary Tract Infections--Preliminary Report. Adv Clin Exp Med. 2015;24(3):441-445.

3. Washington AE, Johnson RE, Sanders LL Jr. Chlamydia trachomatis infection in the United States: what are they costing us? JAMA. 1987; 257(15): 20702072.

4. Martínez T MA, Reid S I, Arias C, Napolitano R C, Sandoval Z J, Molina C R. Prevalencia de infección cervical por Chlamydia trachomatis en mujeres de la Región Metropolitana. Rev Med Chile. 2008; 136(10): 1294-300.

5. Rasmussen SJ, Eckmann L, Quayle AJ, Shen L, Zhang Y X, et al. Secretion of proinflammatory cytokines by epithelial cells in response to Chlamydia infection suggests a central role for epithelial cells in chlamydial pathogenesis. J Clin Invest. 1997; 99(1): 77-87.
6. Paavonen J, Eggert-Kruse W. Chlamydia trachomatis: impact on human reproduction. Hum Reprod Updat. 1999; 5(5): 433-447.

7. Silva R, León D, Viscarra T, lli C, Roa JC, et al. Frecuencia de la infección por Chlamydia trachomatis en un grupo de mujeres de la Región de la Araucanía, Chile. Rev Chilena Infectol. 2013; 30 (6): 611-615.

8. Nassar FA, Abu-Elamreen FH, Shubair ME, Sharif FA. Detection of Chlamydia trachomatis and Mycoplasma hominis, genitalium and Ureaplasma urealyticum by polymerase chain reaction in patients with sterile pyuria. Adv Med Sci. 2008;53(1):80-86.

9. Pedrosa AF, Azevedo F, Lisboa C. Screening for Chlamydia infection in a sexually transmitted infection clinic: a missed opportunity? Int J Dermatol. 2015;54(4):405-409.

10. Roth AM, Rosenberger JG, Reece M, Van Der Pol B. Expanding sexually transmitted infection screening among women and men engaging in transactional sex: the feasibility of field-based self-collection. Int J STD AIDS. 2013;24(4):323-328.

11. Clayton HB, Lowry R, August E, Everett Jones S. Nonmedical Use of Prescription Drugs and Sexual Risk Behaviors. Pediatrics. 2016;137(1):110.

12. Bazan JA, Carr Reese P, Esber A, Lahey S, Ervin M, et al. High prevalence of rectal gonorrhea and Chlamydia infection in women attending a sexually transmitted disease clinic. J Womens Health (Larchmt). 2015 Mar;24(3):182-189.

13. van Liere GA, Hoebe CJ, Dukers-Muijrers NH. Evaluation of the anatomical site distribution of chlamydia and gonorrhoea in men who have sex with men and in high-risk women by routine testing: cross-sectional study revealing missed opportunities for treatment strategies. Sex Transm Infect. 2014;90(1):58-60.

14. Koedijk FD, van Bergen JE, Dukers-Muijrers NH, van Leeuwen AP, Hoebe $\mathrm{CJ}$, et al. The value of testing multiple anatomic sites for gonorrhoea and chlamydia in sexually transmitted infection centres in the Netherlands, 2006-2010. Int J STD AIDS. 2012;23(9):626-631.

15. van Liere GA, Hoebe CJ, Niekamp AM, Koedijk FD, Dukers-Muijrers NH. Standard symptom- and sexual history-based testing misses anorectal Chlamydia trachomatis and neisseria gonorrhoeae infections in swingers and men who have sex with men. Sex Transm Dis. 2013 Apr;40:285-9.

16. Peters RP, Nijsten N, Mutsaers J, Jansen CL, Morré SA, et al. Screening of oropharynx and anorectum increases prevalence of Chlamydia trachomatis and Neisseria gonorrhoeae infection in female STD clinic visitors. Sex Transm Dis. 2011;38:783-787.

17. West SK, Moncada J, Munoz B, Mkocha H, Storey P, et al. Is there evidence for resistance of ocular Chlamydia trachomatis to azithromycin after mass treatment for trachoma control? J Infect Dis. 2014;210(1):65-71.

18. Ljubin-Sternak S, Mestrovic T, Vilibic-Cavlek T, Mlinaric-Galinovic G, Sviben $M$, et al. In vitro susceptibility of urogenital Chlamydia trachomatis strains in a country with high azithromycin consumption rate. Folia Microbiol (Praha). 2013;58(5):361-365. 